\title{
Satellite monitoring of different vegetation types by differential optical absorption spectroscopy (DOAS) in the red spectral range
}

\author{
T. Wagner, S. Beirle, T. Deutschmann, M. Grzegorski, and U. Platt \\ Institut für Umweltphysik, University of Heidelberg, Heidelberg, Germany \\ Received: 29 May 2006 - Published in Atmos. Chem. Phys. Discuss.: 16 August 2006 \\ Revised: 7 November 2006 - Accepted: 18 December 2006 - Published: 10 January 2007
}

\begin{abstract}
A new method for the satellite remote sensing of different types of vegetation and ocean colour is presented. In contrast to existing algorithms relying on the strong change of the reflectivity in the red and near infrared spectral region, our method analyses weak narrow-band (few $\mathrm{nm})$ reflectance structures (i.e. "fingerprint" structures) of vegetation in the red spectral range. It is based on differential optical absorption spectroscopy (DOAS), which is usually applied for the analysis of atmospheric trace gas absorptions. Since the spectra of atmospheric absorption and vegetation reflectance are simultaneously included in the analysis, the effects of atmospheric absorptions are automatically corrected (in contrast to other algorithms). The inclusion of the vegetation spectra also significantly improves the results of the trace gas retrieval. The global maps of the results illustrate the seasonal cycles of different vegetation types. In addition to the vegetation distribution on land, they also show patterns of biological activity in the oceans. Our results indicate that improved sets of vegetation spectra might lead to more accurate and more specific identification of vegetation type in the future.
\end{abstract}

\section{Introduction}

Vegetation has a strong influence on the cycles of trace gases and other important properties of the earth system, in particular the earth's energy budget. Vegetation modifies the ground albedo and thus has a strong impact on the amount of backscattered solar energy. Vegetation also strongly influences the water cycle through its influence on evaporation; the release of latent heat is important for the latitudinal energy distribution. Plants are also sources and/or sinks for many important trace gases, in particular greenhouse gases.
Therefore, the precise knowledge of the spatio-temporal variation of the biological activity is an important prerequisite for the correct understanding and simulation of global trace gas budgets and of the earth's climate. Of special importance is the monitoring of the human-induced change of the global vegetation patterns, e.g. caused by biomass burning or climate change.

Algorithms for the remote sensing of vegetation have been developed and are successfully applied to satellite observations for a long time. Typically they are based on the measured radiance in the red and near-infrared part of the spectrum. Over this wavelength range, the reflectivity of vegetation changes strongly (Fig. 1), caused by the absorption of various kinds of chlorophyll and pigments. Thus a clear vegetation signal can be easily derived. Definitions of various kinds of vegetation indices based on intensity ratios can be found e.g. in Birth and McVey (1968), Jordan (1969), Rouse et al. (1974), Huete (1988), Gutman (1991), and Jensen (2000).

Here we present a new vegetation algorithm which can be applied to new satellite sensors with moderate spectral resolution (but presently only coarse spatial resolution). A similar method was already applied to airborne measurements by Clark et al. (1995). In contrast to the existing algorithms, our method exploits the narrow-band spectral information of the vegetation reflectance, which allows in particular to discriminate different types of vegetation. One additional advantage is that the influence of atmospheric absorption is automatically corrected. These findings are in agreement with the formulation of the forward model for the performed measurements; they are also confirmed by the analysis of simulated spectra.

Correspondence to: T. Wagner

(thomas.wagner@iup.uni-heidelberg.de)

Published by Copernicus GmbH on behalf of the European Geosciences Union. 


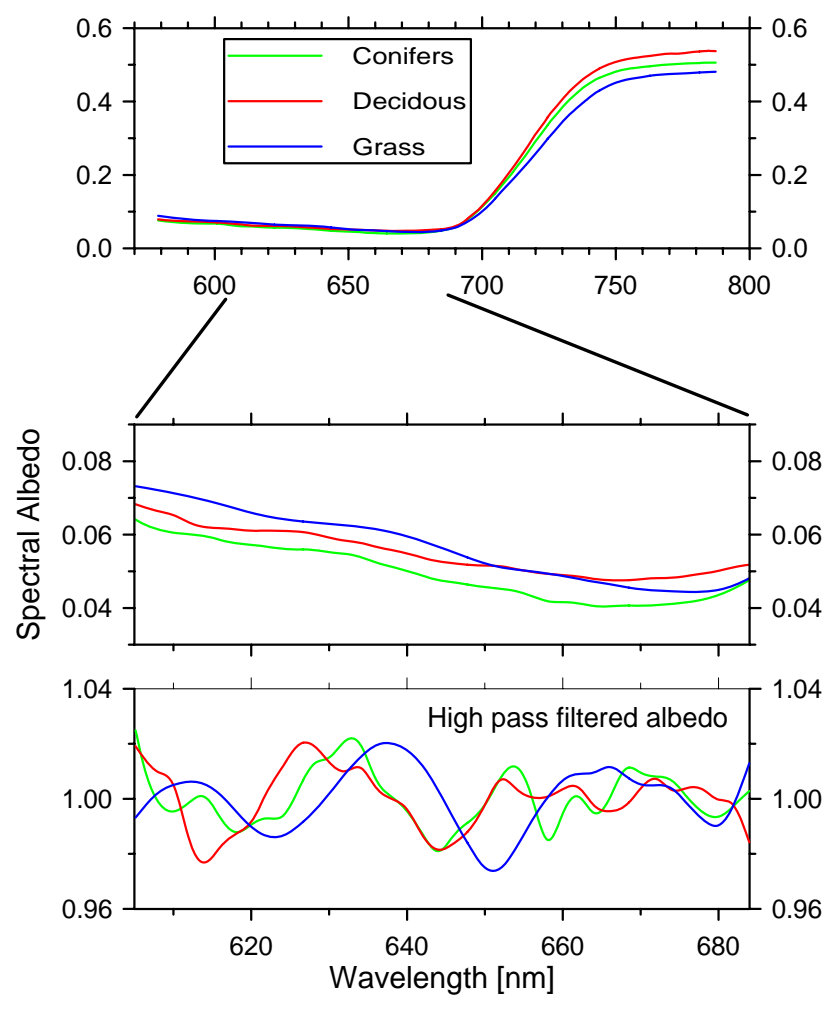

Fig. 1. Top: Spectra of the reflectance over different kinds of vegetation, reproduced from the ASTER Spectral Library through the courtesy of the Jet Propulsion Laboratory, California Institute of Technology, Pasadena, California. (C1999, California Institute of Technology. ALL RIGHTS RESERVED. The strong change of the reflectance between the red and infrared part of the spectrum is usually exploited for the remote sensing of vegetation. In the red part of the spectrum the reflectance is small (middle), but contains characteristic spectral structures. These structures become more obvious after high-pass filtering (division by a fitted polynomial of degree 4) (bottom).

\section{The instrument: GOME on ERS-2}

The Global Ozone Monitoring Experiment (GOME) is one of several instruments aboard the European research satellite ERS-2 (European Space Agency (ESA), 1995; Burrows et al., 1999). It consists of a set of four spectrometers that simultaneously measure sunlight reflected from the Earth's atmosphere and surface in 4096 spectral channels covering the wavelength range between 240 and $790 \mathrm{~nm}$ with moderate spectral resolution (full width at half maximum (FWHM): $0.2-0.4 \mathrm{~nm})$. The satellite operates in a nearly polar, sunsynchronous orbit at an altitude of $780 \mathrm{~km}$ with an equator crossing time of approximately 10:30 a.m. local time. While the satellite orbits in an almost north-south direction, GOME scans the surface of earth in the perpendicular east-west direction. During one scan, three individual ground pixels are observed, each covering an area of $320 \mathrm{~km}$ east to west by $40 \mathrm{~km}$ north to south. They lie side by side: a west, a cen- tre, and an east pixel. The Earth's surface is entirely covered within 3 days, and poleward from about $70^{\circ}$ latitude within 1 day.

\section{Data analysis}

\subsection{Analysis of the measured spectra}

We retrieve information on vegetation and atmospheric absorbers using Differential Optical Absorption Spectroscopy (DOAS, see Platt, 1994). The DOAS method applies a highpass filtering to the measured spectra of (in our case: scattered and reflected sun light) and thus enables the detection of several weak atmospheric absorbers. Our DOAS analysis is performed in the wavelength interval $605-683 \mathrm{~nm}$. It is based on the algorithm developed for the analysis of the atmospheric absorptions of water vapour and the oxygen molecule $\left(\mathrm{O}_{2}\right)$ and dimer $\left(\mathrm{O}_{4}\right)$ as described in detail in Wagner et al. (2004, 2005). Using this algorithm, however, it turned out that over the continents often strong spectral structures appeared in the measured spectra, which could not be accounted for by the atmospheric absorptions of $\mathrm{O}_{2}, \mathrm{O}_{4}$, and $\mathrm{H}_{2} \mathrm{O}$. These spectral structures showed up in the residual of the DOAS analysis causing strong systematic errors of the trace gas retrievals. In particular the $\mathrm{O}_{4}$ absorptions are affected, which have a similar band width as the observed residual structure. In some cases, even apparent negative $\mathrm{O}_{4}$ absorptions were found (Figs. 2 and 3). In contrast, the absorptions of $\mathrm{O}_{2}$ and $\mathrm{H}_{2} \mathrm{O}$ are only weakly affected.

After various putative instrumental and methodological reasons for this structure were investigated and could be excluded, we studied whether spectral structures caused by the albedo of specific surface types might be responsible for the observed spectral residuals. Since the problems occurred only over areas with vital vegetation, we took a closer look at the spectral albedos of different kinds of vegetation (vegetation reflectance spectra reproduced from the ASTER Spectral Library through the courtesy of the Jet Propulsion Laboratory, California Institute of Technology, Pasadena, California, see also http://speclib.jpl.nasa.gov/).

It soon turned out that the residual spectral structures were similar to the high-pass-filtered reflectance spectra measured over vegetation (Fig. 1). Moreover, if the (logarithm of the) vegetation spectra were included in the spectral analysis, the residual structures and the errors for the retrieval of the atmospheric absorbers were strongly reduced (Fig. 2). In particular, the $\mathrm{O}_{4}$ absorptions retrieved in the red spectral range become very similar to those retrieved in the UV (Fig. 3).

It is interesting to note that in the red spectral range not only the absolute values of the albedo, but also the narrowband spectral structures (for the spectral resolution of up to $8 \mathrm{~nm}$, see http://speclib.jpl.nasa.gov/) are very weak $(<3 \%)$. For the corresponding weak variations of the observed radiance, it is thus possible to include the (logarithm of the) 


\section{Spectral fit without vegetation spectra}

27.7.1998, 3:54 UTC, lat: $64.7^{\circ} \mathrm{N}$, long: $117.5^{\circ} \mathrm{E}, \mathrm{SZA}: 45.6^{\circ}$
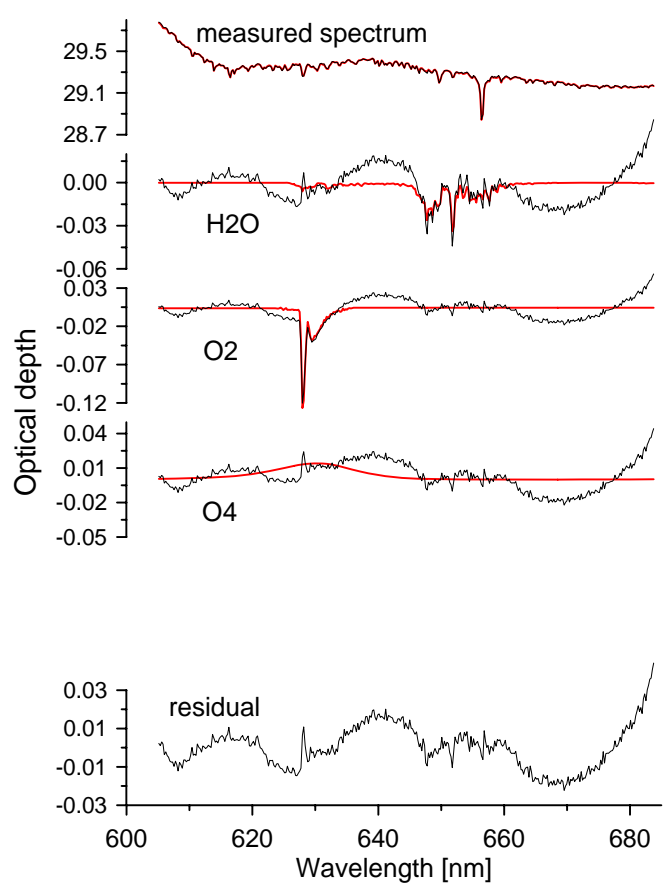

Spectral fit with vegetation spectra

27.7.1998, 3:54 UTC, lat: $64.7^{\circ} \mathrm{N}$, long: $117.5^{\circ} \mathrm{E}, \mathrm{SZA}: 45.6^{\circ}$

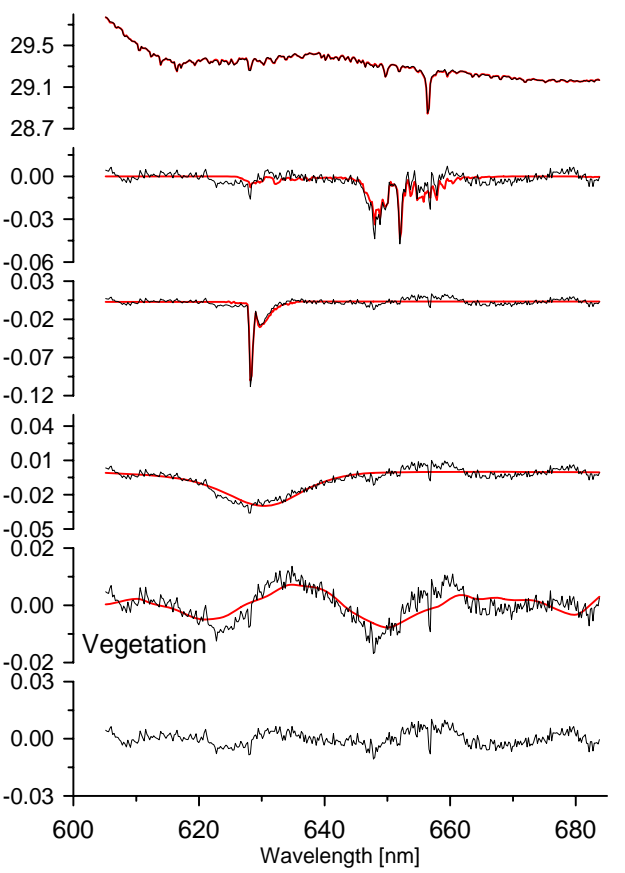

Fig. 2. Results of a spectral DOAS analysis of water vapor and the oxygen molecule $\left(\mathrm{O}_{2}\right)$ and dimer $\left(\mathrm{O}_{4}\right)$ without (left) and with (right) inclusion of vegetation reflectance spectra. The red curves represent the trace gas cross sections and the vegetation spectra scaled to the respective spectral structures detected in the satellite spectrum (black curves). For measurements over vital vegetation always strong and systematic spectral residuals appear, if the reflectance spectra of vegetation are not included.

vegetation spectra (like the trace gas reference spectra) directly in the DOAS fitting procedure (for more details see Sect. 3.2). One particular advantage of the simultaneous fitting of atmospheric trace gas absorptions and spectral albedo structures is that the correction of atmospheric absorption processes that is necessary (and often problematic) for conventional algorithms is automatically included in the retrieval of the vegetation results.

It should be noted that even if the vegetation spectra were included in the fitting procedure, still systematic spectral structures in the residual remained (Fig. 2). Also, the retrieved values for the different kinds of vegetation are sometimes below zero (Fig. 3). These negative values and the remaining residual structures might be caused by several reasons, mainly by a possibly inappropriate spectral resolution and wavelength calibration of the vegetation spectra. We investigated these potential error sources by modifying the DOAS analysis: if e.g. the spectral resolution of the GOME measurements and of the trace gas reference spectra were mathematically reduced before the fitting process, the absolute values of the retrieved results for the different vegetation types became systematically smaller. In fact, the spectral resolution of the vegetation spectra is much coarser than the spectral resolution of the GOME instrument (FWHM about $8 \mathrm{~nm}$ compared to about $0.4 \mathrm{~nm}$ ). However, the residual structure did not substantially change. Thus, it is probable that the coarse resolution of the vegetation spectra is not the sole reason for the remaining residual structures. In a second modified DOAS analysis, we allowed spectral shifting of the vegetation spectra during the fitting procedure, but the results did not change substantially. Part of these findings are also confirmed by the results using synthetic spectra (Sect. 3.3).

In addition to possible spectroscopic shortcomings, the residual structures might be also partly caused by the fact that the selected vegetation spectra are not fully representative for the measured spectral structures of vegetation (also non-vegetation surfaces might cause spectral structures). Indications for this possibility are also found from the comparison of the results for the different vegetation types. Especially, the observed interferences between the different vegetation types and also with the ocean colour indicate that the used vegetation spectra did not perfectly fit to the observed spectral structures. We also found that the results are sensitive to variations of the wavelength range under consideration. Such a dependence is a particular indicator for an imperfect agreement of the spectral structures of the measured and the fitted spectra (see Sect. 3.3). Differences between 


\subsubsection{8 (GOME orbit 81023092)}

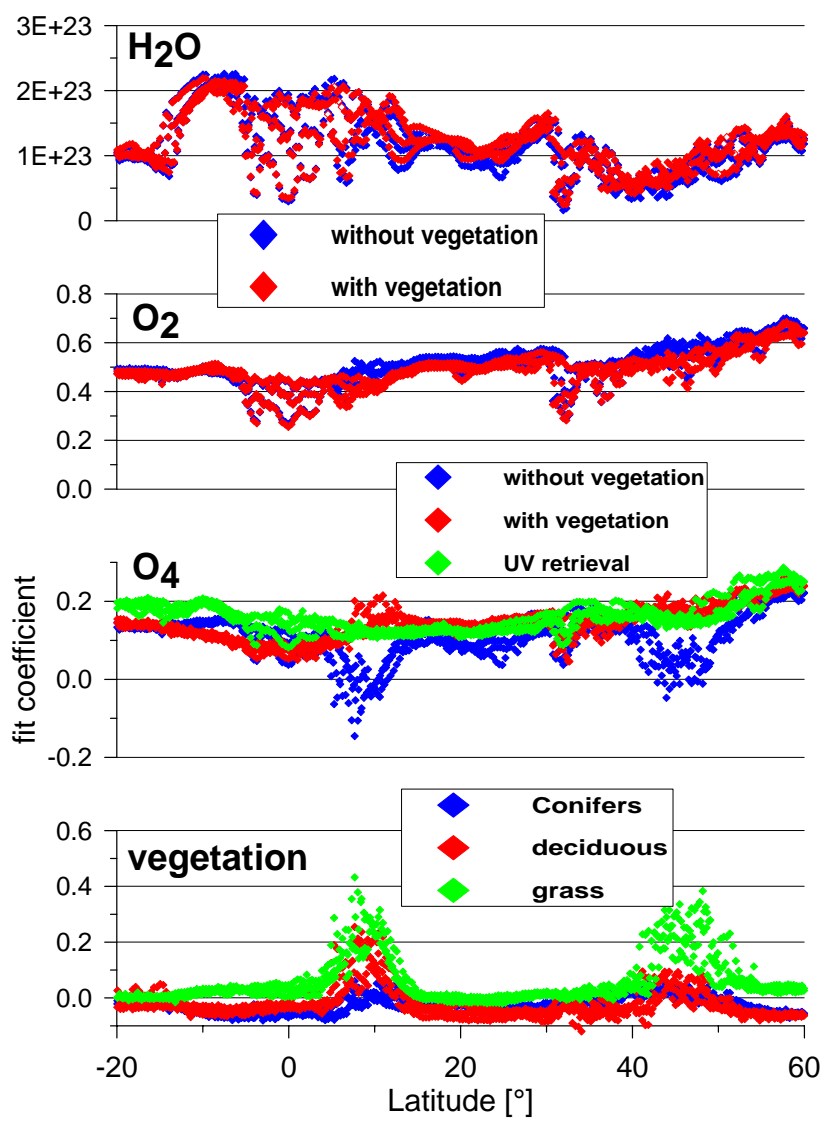

Fig. 3. Results of the absorptions of gaseous atmospheric constituents $\left(\mathrm{H}_{2} \mathrm{O}, \mathrm{O}_{2}, \mathrm{O}_{4}\right.$, expressed in arbitrary units) and (the logarithm of) three vegetation spectra (bottom) for part of one GOME orbit. If no vegetation spectra are included, the $\mathrm{O}_{4}$ absorptions retrieved in the red spectral range differ strongly from those retrieved in the UV for regions with vegetation. In particular they can even become negative. If vegetation spectra are included, the $\mathrm{O}_{4}$ absorption retrieved in the red spectral range become very similar to those retrieved in the UV. The absorptions of $\mathrm{H}_{2} \mathrm{O}$ and $\mathrm{O}_{2}$ are only weakly affected by the inclusion of the vegetation spectra. The enhanced vegetation signal is found over Europe and Africa.

the vegetation spectra and the satellite observations might be also partly caused by the fact that both observations were performed with a different field of view and under different angles of observation and solar illumination.

3.2 Forward model for the influence of a spectral albedo on the DOAS retrieval

To better understand how a spectrally varying ground albedo influences the results of a DOAS analysis, we formulated a forward model describing our observations. One important outcome of this model is that including (the logarithm of) vegetation spectra in a DOAS analysis indeed corrects for the spectral structures in the observed light and also yields direct information on the vegetation coverage of the observed ground pixel. Moreover, our model also allows to determine (and correct for) the sensitivity of the method for various atmospheric conditions.

The measured radiance is either reflected at the Earth's surface or scattered by molecules and particles (aerosols and clouds). On its way through the atmosphere (before and after the reflection or scattering events) the radiance is also attenuated by absorption (by molecules or aerosols).

$I(\lambda)=I_{0}(\lambda) \times e^{-\tau_{\text {refl }}(\lambda)} \times A(\lambda)+I_{\text {scatt }}(\lambda) \times e^{-\tau_{\text {scatt }}(\lambda)}(1)$

Here $I(\lambda)$ is the measured radiance, $I_{o}(\lambda)$ is the radiance which would be measured over a white surface without atmospheric scattering and absorption, $A(\lambda)$ is the surface albedo, $I_{\text {scatt }}(\lambda)$ is the radiance which would be measured over a black surface $(A(\lambda)=0)$ without atmospheric absorption, $\tau_{\text {refl }}(\lambda)$ is the optical depth of the absorption for the reflected photons on their path from the top of the atmosphere (TOA) to the surface and back to TOA, and $\tau_{\text {scatt }}(\lambda)$ is the effective optical depth of the absorption for the scattered photons between TOA, the scattering event (or multiple events) and TOA. To simplify our model, we ignore all photons which underwent both scattering and surface reflection. Because of the low probability for Rayleigh-scattering (in the red spectral range) and the low surface albedo, such photon paths are very rare. Nevertheless, ignoring them leads to a slight underestimation of the sensitivity of a satellite observation to the spectral signature of the surface. Especially in situations with high aerosol concentrations or clouds, such photon paths become more likely.

In the following we will neglect the absorption due to aerosols and molecules, because it is weak (in the considered wavelength range typically a few percent) and/or it is "removed" in the DOAS analysis by fitting the appropriate absorption cross section. With this simplification, Eq. (1) reduces to:

$I(\lambda)=I_{0}(\lambda) \times A(\lambda)+I_{\text {scatt }}(\lambda)$

Now we split the surface albedo into a part with a broad band wavelength dependence and a part with a narrow wavelength dependence:

$A(\lambda)=A(\lambda)_{\text {broad }} \times A(\lambda)_{\text {narrow }}$

This separation can be e.g. done by fitting a low order polynomial to the albedo spectrum. This polynomial then represents $A(\lambda)_{\text {broad }}$ and the ratio of the original albedo spectrum and the polynomial represents $A(\lambda)$ narrow (it should be noted that $A(\lambda)_{\text {narrow }}$ varies around unity (see Fig. 1), in contrast to the usual high-pass-filtered absorption cross sections used in DOAS analyses which vary around zero).

Equation (2) then becomes

$I(\lambda)=I_{0}(\lambda) \times A(\lambda)_{\text {broad }} \times A(\lambda)_{\text {narrow }}+I_{\text {scatt }}(\lambda)$ 
In the DOAS analysis the logarithm of the ratio of an intensity including the narrow band albedo and the intensity without the narrow band albedo effect is calculated:

$\ln \frac{I_{\text {narrow }}(\lambda)}{I_{\text {broad }}(\lambda)}=\ln \frac{I_{0}(\lambda) \times A_{\text {broad }}(\lambda) \times A_{\text {narrow }}(\lambda)+I_{\text {scatt }}(\lambda)}{I_{0}(\lambda) \times A_{\text {broad }}(\lambda)+I_{\text {scatt }}(\lambda)}(5)$

Since the variations of the narrow band albedo spectra are weak $(<3 \%)$, we can use the approximation:

$\ln (1+x) \approx x$

and derive:

$A(\lambda)_{\text {narrow }} \approx \ln A_{\text {narrow }}(\lambda)+1$

Then Eq. (5) becomes:

$\ln \frac{I_{\text {narrow }}(\lambda)}{I_{\text {broad }}(\lambda)}=$

$\ln \frac{I_{0}(\lambda) \times A_{\text {broad }}(\lambda)+I_{\text {scatt }}(\lambda)+I_{0}(\lambda) \times A_{\text {broad }}(\lambda) \times \ln A_{\text {narrow }}(\lambda)}{I_{0}(\lambda) \times A_{\text {broad }}(\lambda)+I_{\text {scatt }}(\lambda)}$

Since the term $I_{0}(\lambda) \times A_{\text {broad }}(\lambda) \times \ln A_{\text {narrow }}(\lambda)$ is small compared to $I_{0}(\lambda) \times A_{\text {broad }}(\lambda)+I_{\text {scatt }}(\lambda)$, we can approximate Eq. (8) by:

$$
\approx \frac{I_{0}(\lambda) \times A_{\text {broad }}(\lambda)}{I_{0}(\lambda) \times A_{\text {broad }}(\lambda)+I_{\text {scatt }}(\lambda)} \times \ln A_{\text {narrow }}(\lambda)
$$

From Eq. (9) we conclude that if we include the logarithm of the narrow-band albedo spectrum in the DOAS analysis, the determined fitting coefficient represents the vegetation cover of the observed ground pixel weighted by the ratio of light reflected at the surface to the total light (sum of reflected and scattered light).

To determine the sensitivity of the satellite observation on the spectral signature of the albedo, the ratio of the reflected light and the total light can be calculated by radiative transfer models. To derive the actual vegetation cover for the observed ground pixel, the retrieved fitting coefficient for the albedo spectrum has to be scaled according to this ratio. In analogy to the use of the term "air mass factor" in the DOAS analysis of atmospheric trace gases, this ratio could be referred to as "surface factor". For a pure Rayleigh atmosphere, surface albedo of about $5 \%$, and overhead sun it is about 0.7; for larger solar zenith angles (SZA) it decreases because the probability for a photon to be scattered before it reaches the ground, increases (see Fig. 4, top).

\subsection{Sensitivity studies using radiative transfer modelling}

To investigate the sensitivity of the satellite observations of spectral albedo structures in more detail, we calculated and analysed synthetic spectra representing the satellite observations. With this approach it was not only possible to confirm the findings of our observations and of the forward model (Eqs. 1 to 9), but it was also possible to perform various sensitivity studies.
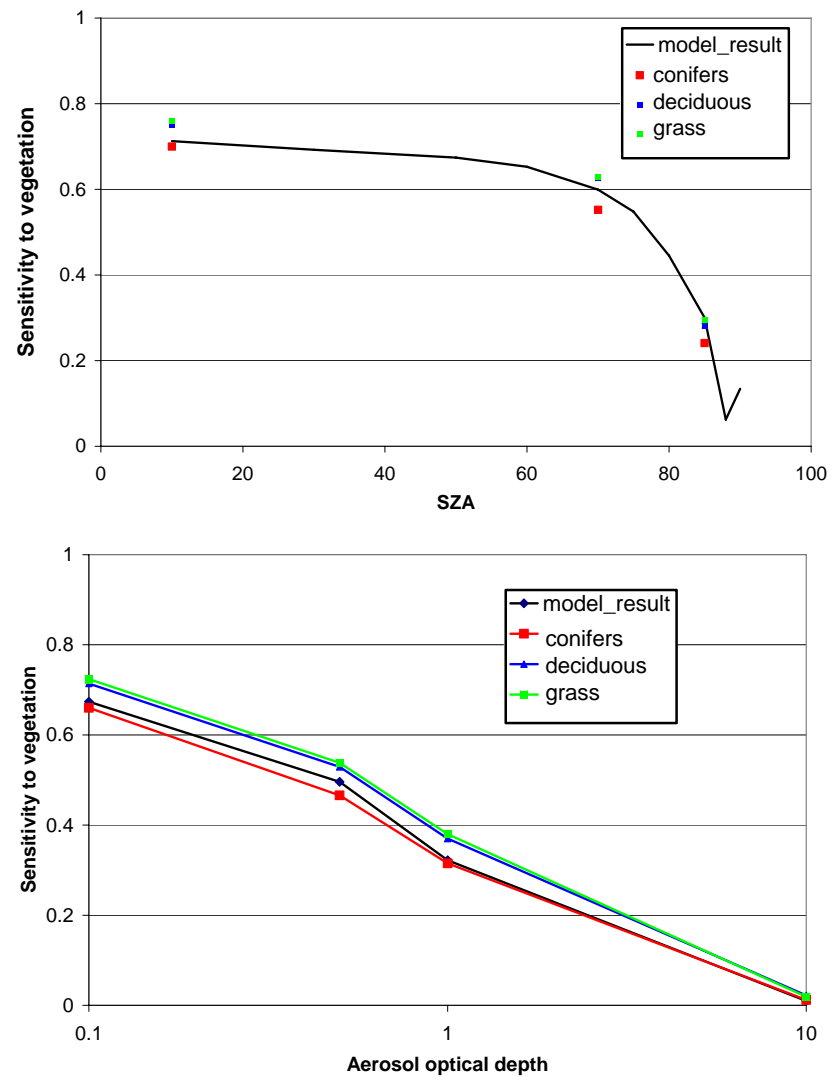

Fig. 4. Top: Sensitivity towards vegetation spectral structures as a function of the solar zenith angle (no aerosols included). The "model results" refer to the ratio of the total radiance to the radiance of only the scattered photons (see Eq. 9). Also displayed are the results derived from the DOAS analysis of the modelled spectra (see Sect. 3.3). Bottom: Sensitivity towards vegetation spectral structures as a function of the aerosols optical depth (SZA: $\left.10^{\circ}\right)$.

The spectra are modelled using our Monte Carlo radiative transfer model TRACY-II (see Deutschmann et al., 2006; Wagner et al., 2006b). Our calculations include the absorptions of the atmospheric oxygen molecule $\left(\mathrm{O}_{2}\right)$ and dimer $\left(\mathrm{O}_{4}\right)$; the surface albedo is treated as a Lambertian reflector using either one of the three vegetation spectra. The modelling of the spectra is performed on a fine wavelength grid $(0.002 \mathrm{~nm})$ to simulate the highly fine structured atmospheric $\mathrm{O}_{2}$ absorption with appropriate spectral resolution. The high resolution spectra are then convoluted by the instrument function of the GOME instrument and sampled according to its dispersion. After forming the logarithm, the trace gas spectra (in this case only those of $\mathrm{O}_{2}$ and $\mathrm{O}_{4}$ ) and the logarithm of the three vegetation spectra are simultaneously fitted to the modelled spectra, exactly in the same way as it was done for the atmospheric measurements.

It should be noted that our simulations include three simplifications to minimise the computational effort: first, we did not consider the wavelength dependence of 

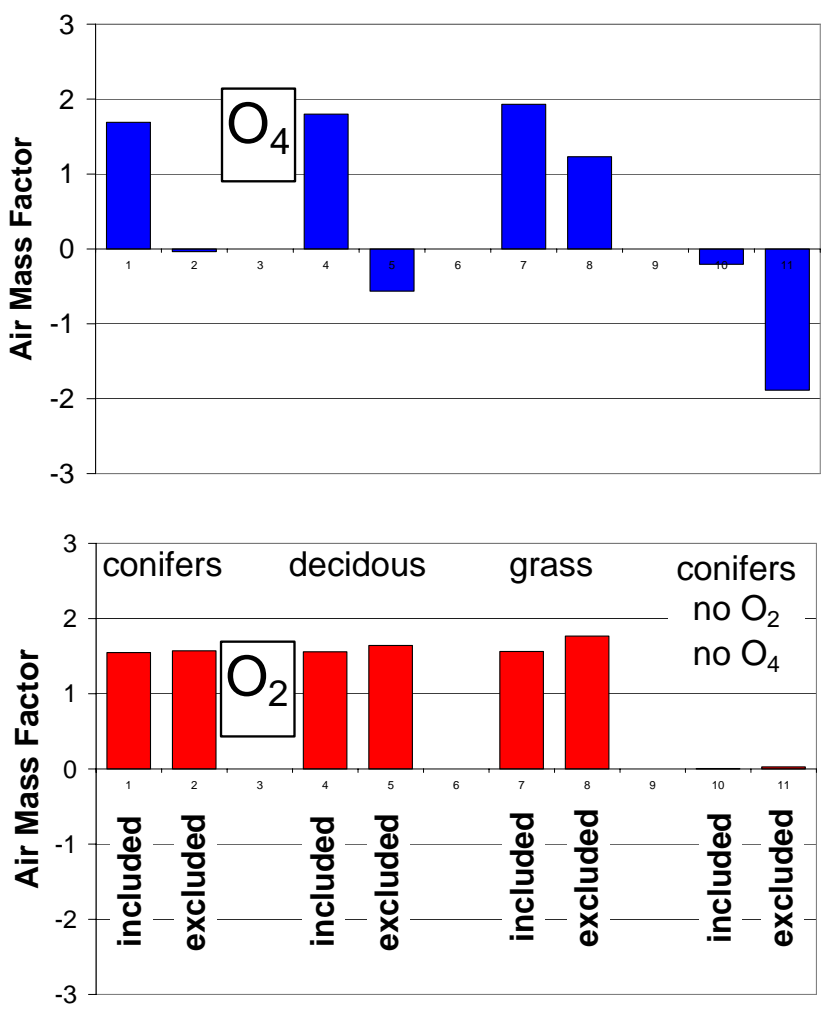

Fig. 5. Trace gas results for the DOAS analysis of the modelled spectra (for a SZA: $10^{\circ}$, without aerosols), expressed as air mass factors (see text). The spectra were modelled for the different vegetation types (conifers, decidous, grass) including the $\mathrm{O}_{2}$ and $\mathrm{O}_{4}$ absorption and for a conifers spectrum without the $\mathrm{O}_{2}$ and $\mathrm{O}_{4}$ absorptions. In the DOAS analysis the vegetation spectra were either included or excluded. The retrieved $\mathrm{O}_{2}$ absorption hardly depends on the inclusion of the vegetation spectra and match the expected values ( $\sim 2$ if the $\mathrm{O}_{2}$ and $\mathrm{O}_{4}$ absorptions are included and 0 if not). In contrast, the retrieved $\mathrm{O}_{4}$ absorption deviates strongly from these values if the vegetation spectra were not considered in the DOAS analysis.

Rayleigh-scattering over the considered wavelength range. From $600 \mathrm{~nm}$ to $680 \mathrm{~nm}$ the decrease of the radiance due to the wavelength dependence of Rayleigh scattering is about $15 \%$ (for a ground albedo of 5\%). This weak broad band spectral dependence can be completely compensated by the polynomial in the DOAS analysis and is thus of no relevance for the performed sensitivity studies.

Second, we did not include the atmospheric $\mathrm{H}_{2} \mathrm{O}$ absorption. Like $\mathrm{O}_{2}$, also the absorptions of $\mathrm{H}_{2} \mathrm{O}$ consists of very many narrow lines. Thus the basic effects of this kind of molecular absorption can be studied already from the modelled $\mathrm{O}_{2}$ absorptions.

Third, we assumed the same relative altitude profile (that of the air density) for the $\mathrm{O}_{4}$ and $\mathrm{O}_{2}$ concentrations. Thus the $\mathrm{O}_{4}$ absorptions retrieved from the synthetic spectra can not be directly compared to the measured $\mathrm{O}_{4}$ absorptions.
Nevertheless, the potential interference effects between the broad band $\mathrm{O}_{4}$ absorption and the vegetation spectra can be studied without any restrictions.

Using a variety of modelled spectra we investigated the sensitivity of our method for various measurement conditions, possible spectroscopic shortcomings of the vegetation spectra, and for different settings of the DOAS fitting parameters. In a first exercise, we determined the sensitivity of the retrieved vegetation results on the solar zenith angle and aerosol scattering. In the simulations we used alternatively each of the three vegetation spectra. In Fig. 4 (top) the fitting coefficients for the (logarithm of) the three vegetation spectra are shown as a function of the solar zenith angle (for an atmosphere without aerosols). The sensitivity to the vegetation spectra is similar for all three vegetation spectra; it is about $70 \%$ for $10^{\circ} \mathrm{SZA}$ and decreases towards larger SZA. The results also agree with the modelled sensitivity which was determined by simply ratioing the radiance which is reflected from the surface by the total radiance (according to Eq. 9).

To simulate the influence of additional scattering due to aerosols and clouds, we modelled synthetic spectra for different optical depths of aerosol scattering (Fig. 4, bottom). As expected from Eq. (9), the sensitivity for the spectral vegetation structures decreases with increasing frequency of aerosol scattering. For an aerosol optical density of 1, the sensitivity is reduced to only about half of that for clear skies, for an optical density of 10 it is almost zero. These findings indicate that for the correct interpretation of the DOAS results for the vegetation spectra, an appropriate cloud and aerosol correction has to be applied. Fortunately, such a correction can be easily performed if the (effective) cloud fraction of a satellite observation is known (e.g. taken the measured absolute radiance as a proxy, or using the Heidelberg iterative cloud retrieval utilities (HICRU [Grzegorski et al., 2006). It is interesting to note, that for such a cloud correction no information on the altitude of the cloud or aerosol layer is necessary (as is needed e.g. for the correction of tropospheric trace gases observations), since in contrast to a trace gas layer, the Earth's surface is always below any cloud or aerosol layer.

In the second exercise we investigated the errors of the trace gas results, which arise if the vegetation spectra are not included in the DOAS analysis. Figure 5 shows the results for the $\mathrm{O}_{2}$ and $\mathrm{O}_{4}$ absorptions for different synthetic spectra with either the vegetation spectra included or excluded. To allow a easy interpretation, the results are expressed as air mass factor (AMF), which is the ratio of the measured absorption and the absorption of vertical atmospheric path (see e.g. Solomon et al., 1987). For a nadir viewing instrument and a solar zenith angle of $10^{\circ}$ the air mass factor is slightly lower than 2 indicating that the majority of the measured photons have traversed the atmosphere twice (if the synthetic spectra do not include the $\mathrm{O}_{2}$ and $\mathrm{O}_{4}$ absorptions, the air mass factor should be 0 ). 

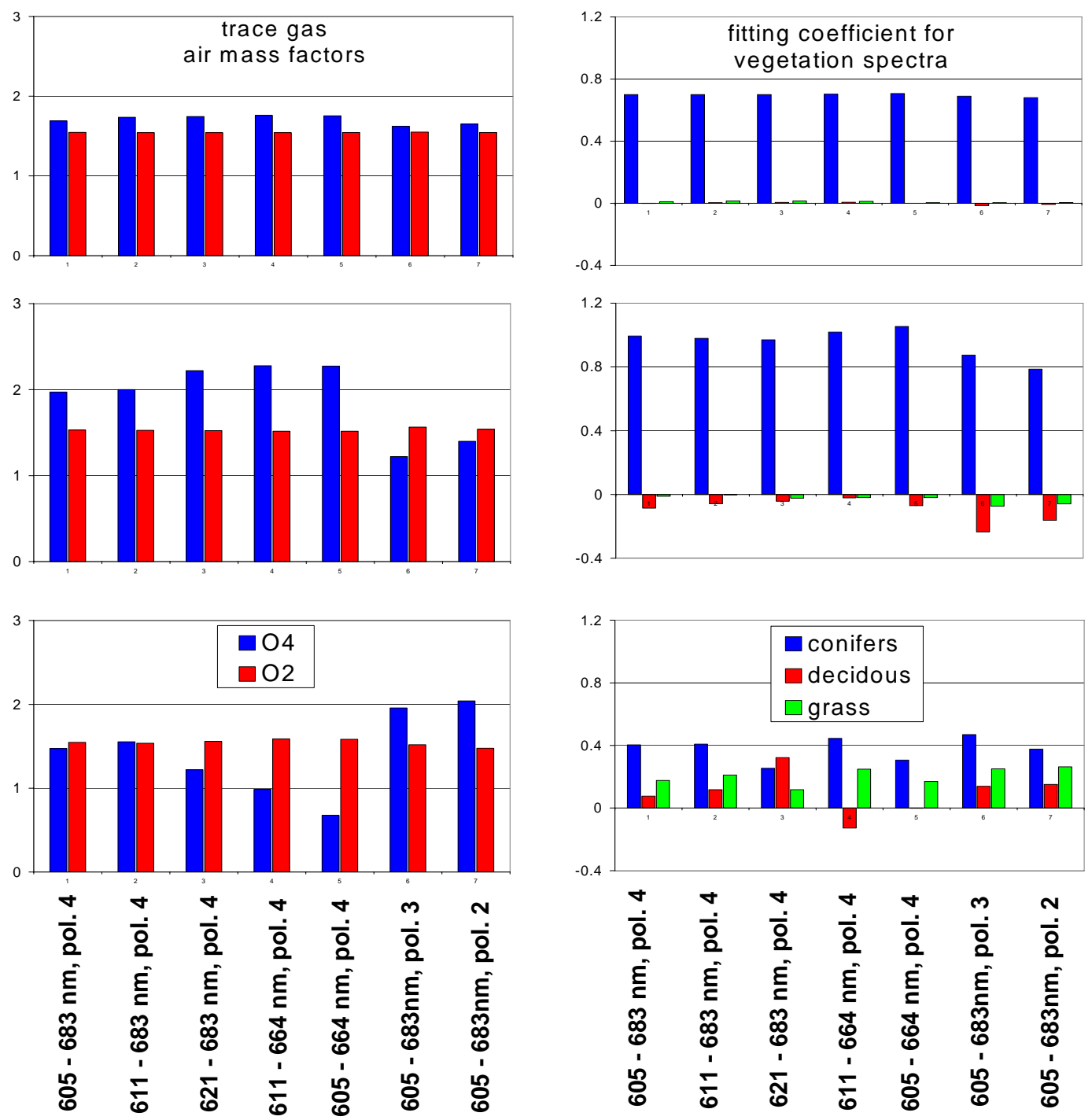

Fig. 6. Results for the DOAS analysis of modelled spectra using the original vegetation spectra (top), using vegetation spectra convoluted by a Gaussian function of $4.6 \mathrm{~nm}$ FWHM (middle), or using vegetation spectra shifted by $-1 \mathrm{~nm}$ (bottom). The spectra were modelled for a SZA: $10^{\circ}$, without aerosols, and for a conifers spectrum as albedo. The different results in the individual graphs are retrieved for different fitting parameters. If the original vegetation spectra are used in the DOAS retrieval, the DOAS results hardly depend on the fitting parameters (top). They also fit the expected values (air mass factor of slightly lower than 2 for the trace gas absorptions; fitting coefficient of about 0.7 for the conifer spectrum, 0 for the other vegetation spectra). If smoothed or shifted vegetation spectra are used, the results differ from the expected values and also strongly depend on the fitting parameters.

Our model results show that only if the vegetation spectra are included, the $\mathrm{O}_{4}$ results match the expected values. If they are excluded, they strongly deviate from the expected values and can become even negative, in agreement with the results of the real measurements (see Figs. 2 and 3). In contrast, the $\mathrm{O}_{2}$ absorptions hardly depend on the inclusion of the vegetation spectra, because the fine-structured absorption only weakly interferes with the vegetation spectra. This finding is also in good agreement with the results of the real observations.
In the third exercise we investigated the sensitivity of the retrieved results on the quality of the vegetation spectra and the fitting parameters of the DOAS analysis. For that purpose we modelled a synthetic spectrum for a SZA of $10^{\circ}$ without aerosols and including the conifers albedo spectrum. The DOAS analysis of this spectrum was performed using different wavelength ranges and different degrees of the polynomial. If the original vegetation spectra are used in the DOAS analysis, the retrieved results match the expected values (the AMF is about 2, the fitting coefficient for 

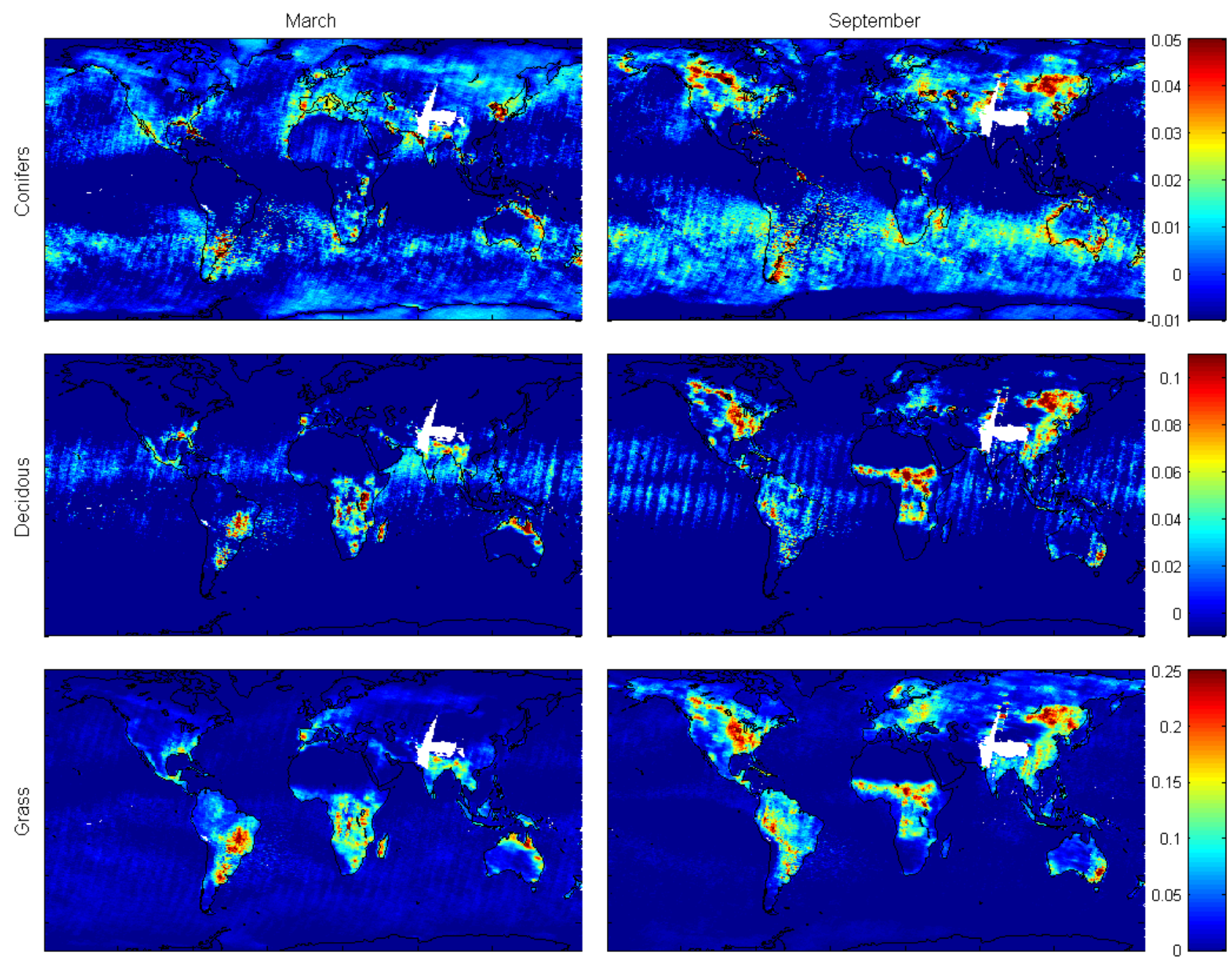

Fig. 7. Global monthly mean results of the DOAS retrieved fitting coefficients for (the logarithms of the) different vegetation spectra (top: conifers, centre: deciduous, bottom: grass). The results for deciduous trees and grass are very similar; those for conifers show different spatial patterns. The effect of the seasonal cycle is clearly visible.

the conifers spectrum is about 0.7 and for the other vegetation spectra it is about 0 ). In particular, the results depend only weakly on the fitting parameters (Fig. 6 top). In two additional analyses we used modified vegetation spectra in the DOAS analysis. First we convoluted them by a Gaussian function with a full width at half maximum (FWHM) of about $4.6 \mathrm{~nm}$ representing the case that the vegetation spectra were measured with an inappropriate low spectral resolution. Second, we shifted the vegetation spectra by $-1 \mathrm{~nm}$ representing the case that the vegetation spectra were sampled on a wrong wavelength grid. For both sets of manipulated vegetation spectra, the retrieved results become strongly dependent on the selected fitting parameters of the DOAS analyses. In addition for most cases, they also deviate from the expected values. It might be interesting to note that the fitting coefficients for the vegetation spectra become systematically smaller, if (instead of the fitted vegetation spectra) the simulated spectra are smoothed, in agreement with the analysis of the real measurements (it should be noted that the analysis of synthetic spectra can not correctly simulate the possible case that the true spectral resolution of the vegetation structures is higher than that of the available vegetation spectra; thus the results of the exercise with smoothed spectra can only give hints on the true effect).

Our results indicate that especially an inaccurate wavelength calibration can lead to large errors, both of the atmospheric trace gas absorptions and the vegetation spectra. To keep the errors of the results of the DOAS analysis $<10 \%$, the accuracy of the wavelength calibration should be better than $0.2 \mathrm{~nm}$ (using modern instrumentation it should be no problem to achieve a better accuracy). 

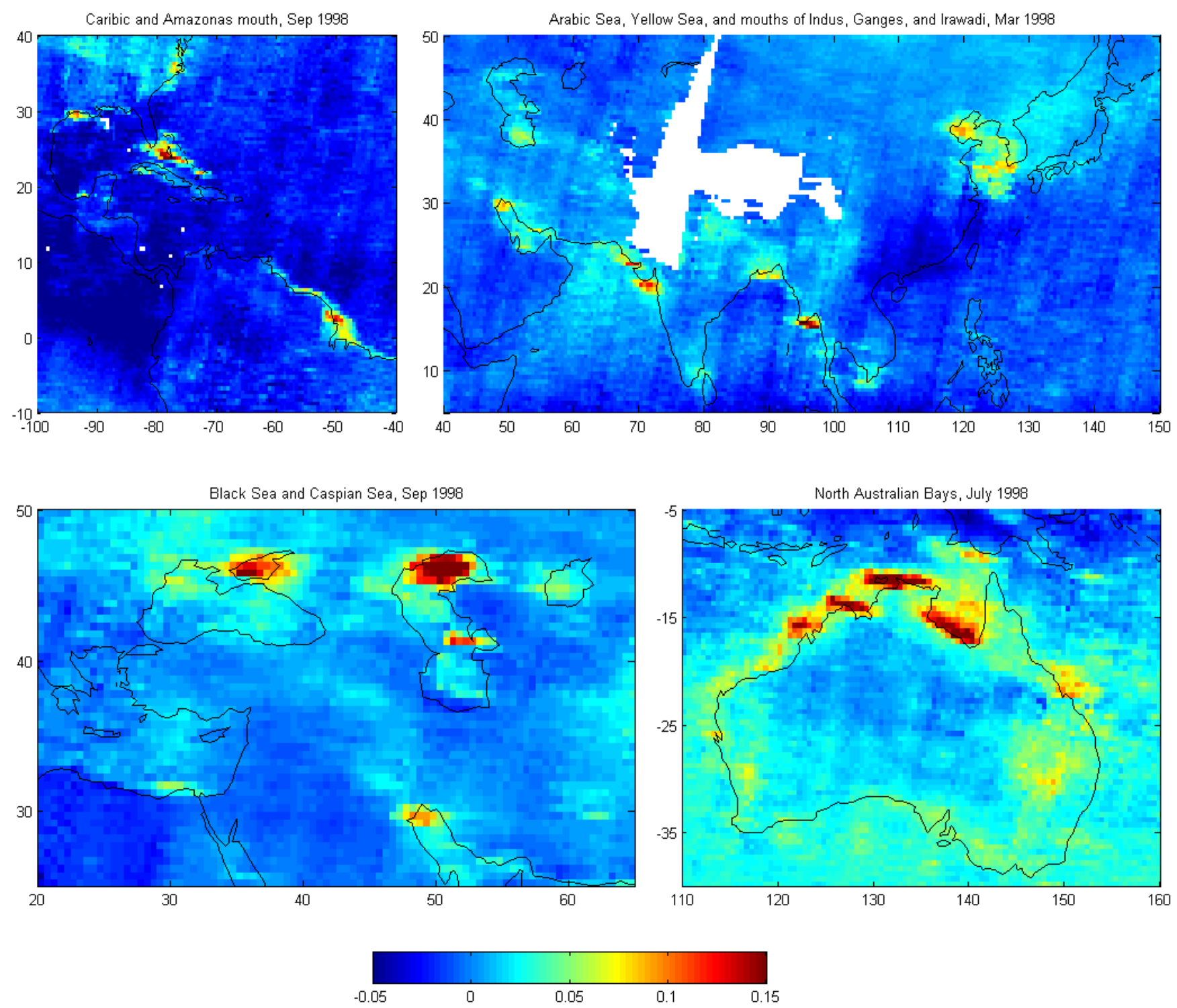

Fig. 8. The retrieved conifer spectral signatures (fitting coefficients for the logarithm of the conifer spectra) are also strongly influenced by the ocean colour. Over areas with strong biological activity, high values are found.

The findings of our sensitivity study indicate that the remaining problems of the DOAS analysis of the satellite measurements (see Sect. 3.1) might be (at least partly) caused by an inappropriate spectral resolution and wavelength calibration of the used vegetation spectra.

\section{Results}

In Fig. 7, monthly mean maps for the results of the different vegetation spectra are shown for two selected months (March and September 1998). Three types of vegetation (conifers, deciduous trees, grass and dry grass) were included in the spectral fitting procedure.
Only measurements with an $\mathrm{O}_{2}$ absorption $>80 \%$ of the maximum $\mathrm{O}_{2}$ absorption were selected to exclude mainly cloudy skies (for details of the cloud-selection algorithm, see Wagner et al., 2005, 2006a). It should be noted that a more accurate cloud correction could probably be achieved using the measured absolute radiance as an indicator for cloud cover (see Sect. 3.3). However, for simplicity, in this study we chose the $\mathrm{O}_{2}$ absorption as indicator for the cloud cover, because the $\mathrm{O}_{2}$ absorption is retrieved simultaneously with the result for the vegetation spectra. In addition, we did not correct the DOAS results for the vegetation spectra for the SZA dependence of the sensitivity (see Fig. 4). For SZA $>80^{\circ}$, the sensitivity decreases rapidly and our data underestimate the true vegetation coverage significantly. 
However, for the GOME observations during the selected months SZA $>80^{\circ}$ occur only for latitudes north and south of $75^{\circ} \mathrm{N}$ and $75^{\circ} \mathrm{S}$, respectively.

The results for the three vegetation spectra show characteristic spatio-temporal patterns. The relative patterns for deciduous trees and grass are almost identical. This is an interesting finding, since both spectra show different narrowband spectral structures (Fig. 1). It indicates that the observed spectral structures of vegetation contain components of both vegetation spectra. For conifers, different patterns were found. Enhanced values are mainly located over the mid and high-latitude regions of the northern hemispheric continents, in good agreement with the global distribution of boreal coniferous forest. It is interesting to note that especially the results for conifers are also influenced by the ocean colour. Enhanced values are found over regions with high biological activity, particularly close to the mouths of big rivers (see Fig. 8). In some of these regions, also coral reefs exist, which might have also contributed to the measured vegetation signal. It should be noted that in this study, no ocean spectra could be included in the analysis because of the typically very low spectral resolution and/or sampling of available ocean spectra.

From our satellite results of vegetation and ocean colour the seasonal cycle of the biological activity in different parts of the world can be clearly followed and similar patterns are found in different years. A comparison to other global data sets of vegetation coverage reveals a good agreement (see e.g. the NOAA Global Vegetation Index Products, available via http://www.osdpd.noaa.gov/PSB/IMAGES/gvi.html). A comprehensive validation is, however, out of scope of this prototype study and should be the subject of future investigations.

The vegetation results can in principle be retrieved on a daily basis; averaging over longer periods of time leads to more consistent patterns, mainly because then the masking due to clouds has a much weaker impact.

\section{Discussion and conclusions}

We included spectra of the (logarithm of the) spectral reflectance for different types of vegetation in the DOAS fitting procedure for the analysis of atmospheric trace gases in the red part of the electromagnetic spectrum. Besides a significant improvement of the fitting results for the atmospheric trace gases, this inclusion enables also the retrieval of vegetation properties from satellite observations. In contrast to the analysis of vegetation indices in the red and near-infrared part of the spectrum (e.g. Jensen, 2000), here we made use of the narrow-band spectral information. In the red part of the spectrum the amplitude of the spectral structures of vegetation is small $(<3 \%)$, but can be clearly identified in the measured spectra. It is in particular possible to identify different kinds of vegetation. Our new method is not only sensitive to vegetation over the continents, but also to the biological activity in the oceans. Especially in the mouths of big rivers and over areas with coral reefs strong vegetation signals can be found. One particular advantage of our new vegetation analysis is that the correction of atmospheric absorption processes is automatically included in the retrieval.

Our results indicate that the currently available vegetation spectra are probably not of sufficient quality to obtain optimum DOAS fitting results, which is understandable since these spectra were of course taken for other purposes. Even if the vegetation spectra are included in the DOAS analysis, the residual still shows remaining systematic structures (see Fig. 2). From our sensitivity studies we conclude that particularly the spectral resolution and the spectral calibration should be improved (accuracy of the wavelength calibration should be better than $0.2 \mathrm{~nm}$ and spectral resolution similar to GOME). In addition, it seems that the selected spectra might not be fully representative for the spectral structures typically observed by satellite sensors. We therefore strongly recommend the measurement of new vegetation spectra covering a larger variety of species including marine algae, different kinds of phytoplankton and coral reefs with better spectral quality. Our results indicate that using this new method it might be possible in the future to monitor the seasonal cycles as well as short term variations of different types of vegetation on a global scale.

Our new vegetation algorithm can also be applied to additional satellite sensors with sufficient coverage of the red spectral region, namely the SCanning Imaging Absorption SpectroMeter for Atmospheric ChartographY (SCIAMACHY) on ENVISAT in Bovensmann et al. (1999), as well as the three instruments of the GOME-II series (EUMETSAT, 2005) (the first instrument has been successfully launched in October 2006). Compared to the GOME-I instrument, the spatial resolution of these sensors is much better (footprints of $30 \times 60 \mathrm{~km}^{2}$ and $40 \times 80 \mathrm{~km}^{2}$, respectively) allowing to retrieve much finer details of the vegetation patterns.

Acknowledgements. The spectra of the vegetation reflectance were reproduced from the ASTER Spectral Library through the courtesy of the Jet Propulsion Laboratory, California Institute of Technology, Pasadena, California. (C)1999, California Institute of Technology. ALL RIGHTS RESERVED, see also http://speclib.jpl.nasa.gov/. Special thanks are expressed to M. Bugert for helpful information on chlorophyll spectra.

Edited by: S. Quegan

\section{References}

Birth, G. S. and McVey, G.: Measuring the color growing turf with a reflectance spectrophotometer, Agronomy J., 60, 640-643, 1968.

Bovensmann, H., Burrows, J. P., Buchwitz, M., Frerik, J., Noël, S., Rozanov, V. V., Chance, K. V., and Goede, A.: SCIAMACHY - 
mission objectives and measurement modes, J. Atmos. Sci., 56, 127-150, 1999.

Burrows, J. P., Weber, M., Buchwitz, M., Rozanov, V., LadstätterWeißenmayer, A., Richter, A., DeBeek, R., Hoogen, R., Bramstedt, K., Eichmann, K.-U., Eisinger, M., and Perner, D.: The Global Ozone Monitoring Experiment (GOME): Mission Concept and First Scientific Results, J. Atmos. Sci., 56, 151-175, 1999.

Clark, R. N., King, T. V. V., Ager, C., and Swayze, G. A.: Initial vegetation species and senescence/stress mapping in the San Luis Calley, Colorado using imaging spectrometer data, Proceedings: Summitville Forum '95, edited by: Posey, H. H., Pendelton, J. A., and Van Zyl, D., Colorado Geological Survey Special Publication, 38, 64-69, 1995.

Deutschmann, T., Wagner, T., and v. Friedeburg, C.: TRACY-II Users manual, University of Heidelberg, 2006.

ESA Publication Division (SP-1182): GOME, Global Ozone Monitoring Experiment, users manual, edited by: Bednarz, F., European Space Research and Technology Centre (ESTEC), Frascati, Italy, 1995.

EUMETSAT: GOME-2 Products Guide, http://www.eumetsat.int/ en/area4/eps/product_guides/GOME-2/GOME2-PG.pdf, 2005.

Grzegorski, M., Wenig, M., Platt, U., Stammes, P., Fournier, N., and Wagner, T.: The Heidelberg iterative cloud retrieval utilities (HICRU) and its application to GOME data, Atmos. Chem. Phys., 6, 4461-4476, 2006, http://www.atmos-chem-phys.net/6/4461/2006/.

Gutman, G. G.: Vegetation indices from AVHRR: An update and future prospects, Rem. Sens. Environ. 35, 121-136, 1991.

Huete, A. R.: A Soil-Adjusted Vegetation Index (SAVI), Rem. Sens. Environ., 25, 295-309, 1988.
Jensen, J. R.: Remote Sensing of the Environment: An Earth Resource Respective, Prentice Hall, Upper Saddle River, New Jersey, 353, 2000.

Jordan, C. F.: Derivation of leaf-area index from quality of light on the forest floor, Ecology, 50(4), 663-666, 1969.

Platt, U.: Differential optical absorption spectroscopy (DOAS), Air monitoring by spectroscopic techniques, edited by: Sigrist, M. W., Chemical Analysis Series, 127, John Wiley \& Sons, Inc, 1994.

Rouse, J. W., Haas, R. H., Schell, J. A., and Deering, D. W.: Monitoring Vegetation Systems in the Great Plains with ERTS, Proceedings, Third Earth Resources Technology Satellite-1 Symposium, Greenbelt: NASA SP-351, 3010-3017, 1974.

Solomon, S., Schmeltekopf, A. L., and Sanders, R. W.: On the interpretation of zenith sky absorption measurements, J. Geophys. Res, 92, 8311-8319, 1987.

Wagner, T., Beirle, S., Grzegorski, M., Sanghavi, S., and Platt, U.: El-Niño induced anomalies in global data sets of water vapour and cloud cover derived from GOME on ERS-2, J. Geophys. Res., 110, D15104, doi:10.1029/2005JD005972, 2005.

Wagner, T., Beirle, S., Grzegorski, M., and Platt, U.: Global trends (1996 to 2003) of total column precipitable water observed by GOME on ERS-2 and their relation to surface-near temperature, J. Geophys. Res., 111, D12102, doi:10.1029/2005JD006523, 2006a.

Wagner, T., Burrows, J. P., Deutschmann, T., et al.: Comparison of Box-Air-Mass-Factors and Radiances for Multiple-Axis Differential Optical Absorption Spectroscopy (MAX-DOAS) Geometries calculated from different UV/visible Radiative Transfer Models, Atmos. Chem. Phys. Discuss., 6, 9823-9876, 2006 b. 